\title{
MicroRNA-145-loaded poly(lactic-co-glycolic acid) nanoparticles attenuate venous intimal hyperplasia in a rabbit model
}

Hiroomi Nishio, MD, Hidetoshi Masumoto, MD, PhD, Kazuhisa Sakamoto, MD, Kazuhiro Yamazaki, MD, PhD, Tadashi Ikeda, MD, PhD, and Kenji Minatoya, MD, PhD

\section{ABSTRACT}

Background: MicroRNA-145 (miR-145) reportedly alters the phenotype of vascular smooth muscle cells (VSMCs) from a proliferative to a contractile state. So far, viral or plasmid vectors have been experimentally used to transduce microRNAs into VSMCs. We hypothesized that a simple ex vivo microRNA delivery system using miR-145-loaded poly(lactic-co-glycolic acid) (PLGA) nanoparticles (PLGA NPs) could control the VSMC phenotype and prevent intimal hyperplasia.

Methods: Jugular vein grafts of male Japanese white rabbits were soaked in phosphate-buffered saline, control microRNA (cont-miR)-loaded PLGA NP solution or miR-145-loaded PLGA NP solution for 30 minutes ( $n=8$ for each). Vein grafts were implanted in the ipsilateral carotid artery and assessed 2 weeks after the implantation.

Results: Quantitative polymerase chain reaction analysis showed significantly higher miR-145 expression in the miR-145-treated group. The neointimal area was significantly smaller in the miR-145-treated group (phosphate-buffered saline-treated vs cont-miR-treated vs miR-145-treated group; $1.63 \pm 0.52 \mathrm{~mm}^{2}$ vs $1.67 \pm 0.49 \mathrm{~mm}^{2}$ vs $0.88 \pm 0.34 \mathrm{~mm}^{2}$, respectively; $P<.01$ for the miR145 -treated vs the cont-miR-treated group). In the miR-145-treated group, $\mathrm{Ki}$ 67-positive cells were significantly fewer, indicating lower VSMC proliferation. An inflammation-related molecule, CD40 expression was significantly reduced by miR-145-loaded PLGA NP treatment.

Conclusions: Local and sustained release of miR-145 by PLGA NPs attenuated intimal hyperplasia in the rabbit model by maintaining VSMCs in a contractile state. This simple ex vivo miR-145 delivery system would be promising toward broader clinical application. (J Thorac Cardiovasc Surg 2019;157:2242-51)

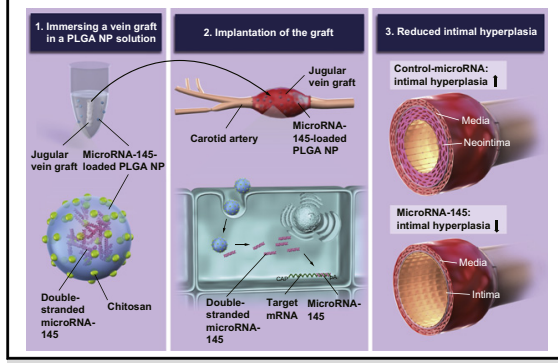

MicroRNA-145-treated vein grafts show significantly lower rate of intimal hyperplasia.

\section{Central Message}

MicroRNA-145-loaded poly(lactic-co-glycolic acid) nanoparticles attenuate graft vein intimal hyperplasia by retaining vascular smooth muscle cells in a contractile phenotype.

\section{Perspective}

Local administration of microRNA-145 might be protective against vein graft disease in patients who undergo coronary or peripheral artery bypass procedures. We proposed a simple method to transduce microRNA-145 by immersing vein grafts into a microRNA-145loaded poly(lactic-co-glycolic acid) nanoparticle solution, which could be widely applied in the clinical setting.

See Commentaries on pages 2252 and 2254.
The saphenous veins have been commonly used as autologous grafts for coronary or peripheral artery bypass graft procedures. However, their long-term postoperative

From the Department of Cardiovascular Surgery, Graduate School of Medicine, Kyoto University, Kyoto, Japan.

This work was supported by research grants from the Ministry of Education, Culture, Sports, Science and Technology, Japan (K.Y.) and a Grants-in-aid of the Cardiovascular Research Fund, Sumitomo Mitsui Trust Bank, Tokyo, Japan (K.S.)

Received for publication May 24, 2018; revisions received Aug 14, 2018; accepted for publication Aug 30, 2018; available ahead of print Nov 14, 2018.

Address for reprints: Hidetoshi Masumoto, MD, PhD, Department of Cardiovascular Surgery, Graduate School of Medicine, Kyoto University, 54 Kawahara-cho, Shougoin, Sakyo-ku, Kyoto 606-8507, Japan (E-mail: masumoto@kuhp.kyoto-u.ac.jp). 0022-5223/\$36.00

Copyright (c) 2018 by The American Association for Thoracic Surgery

https://doi.org/10.1016/j.jtcvs.2018.08.115 patency remains poor. ${ }^{1}$ When exposed to arterial pressure, atherosclerosis of the veins can be accelerated, eventually causing vein graft diseases through intimal hyperplasia. ${ }^{2}$ Intimal hyperplasia is defined as the accumulation of vascular smooth muscle cells (VSMCs) and extracellular matrix in the intima and represents the basis for the later development of graft atheroma. ${ }^{2,3}$ In other words, VSMCs

Scanning this QR code will take you to the article title page to access supplementary information.

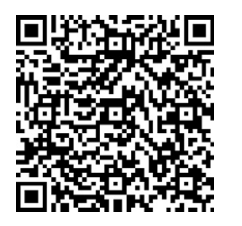




\section{Abbreviations and Acronyms \\ Cont-miR $=$ control microRNA \\ dsRNA $=$ double-stranded RNA \\ FITC = fluorescein isothiocyanate \\ KLF4 = Kruppel-like factor 4 \\ miR-145 = MicroRNA-145 \\ $\mathrm{NF}-\kappa \mathrm{B}=$ nuclear factor-kappa $\mathrm{B}$ \\ PBS = phosphate-buffered saline \\ PCR = polymerase chain reaction \\ PLGA $=$ poly(lactic-co-glycolic acid) \\ PLGA NP = poly(lactic-co-glycolic acid) nanoparticle \\ VSMC = vascular smooth muscle cell}

alter their phenotype from a mature, contractile state to a dedifferentiated, synthetic state and consequently proliferate, migrate from the media into the hyperplastic neointima, and yield extracellular matrices. Numerous basic studies have focused on the control of intimal hyperplasia through the phenotypic control of VSMCs. ${ }^{4}$ Although gene therapy, which introduces genes into targeted cells, has emerged as an encouraging therapeutic modality and has been attempted to regulate intimal hyperplasia in the past 2 decades, ${ }^{5}$ the results of clinical studies are still limited. ${ }^{6}$

MicroRNAs have been widely recognized as a new therapeutic agent in recent years. MicroRNAs consist of 20 to 25 nucleotides and are small noncoding RNAs that can regulate protein expression at the translational level. It is reported that microRNAs play essential roles in various diseases such as cancers or cardiovascular disorders. ${ }^{7,8}$ MicroRNA-145 (miR-145) is expressed in VSMCs and is downregulated in the hyperplastic or atherosclerotic intima of the vascular wall. ${ }^{9}$ We have previously shown that plasmid-based overexpression of miR-145 on a rabbit jugular venous graft reduces neointimal formation by regulating the VSMC phenotype, which required in vivo electroporation. ${ }^{10}$ To circumvent virus- or plasmid-related adverse effects, another simple and effective method for the delivery would be desirable.

Poly(lactic-co-glycolic acid) (PLGA) is a biocompatible and biodegradable material and has been approved by the United States Food and Drug Administration for human clinical uses, such as absorbable surgical sutures. ${ }^{11,12}$ The slow degradation profile of PLGA is ideal for the controlled release of agents and the rate of release can be altered by varying the lactic acid-to-glycolic acid ratio in the copolymer. It is considered that the nanoscale PLGA structures (PLGA nanoparticles; PLGA NPs) are beneficial for efficient cellular uptake via endocytosis. ${ }^{13}$ We hypothesized that miR-145-loaded PLGA NPs could be used to deliver the microRNA to VSMCs of vein grafts and to reduce intimal hyperplasia and attempted to validate the hypothesis using an experimental rabbit model.

\section{METHODS}

Detailed methods are provided in the Online Data Supplement.

\section{Preparation of Lipid-Conjugated Double-Stranded RNAs}

Two double-stranded RNA (dsRNA) sequences were synthesized to be processed in the cytoplasm. These dsRNAs were complexes of the hasmiR-145-5p or control microRNA (cont-miR), consisting of a random sequence of nucleotides, and their complementary oligonucleotides. To overexpress the dsRNAs, a few modifications were made, as reported previously. $^{14-18}$

\section{Preparation of PLGA NPs}

A PLGA copolymer was used as a wall material for nanoparticles with a lactide to glycolide ratio of 75:25. Fluorescein isothiocyanate (FITC)loaded, miR-145-loaded, and cont-miR-loaded PLGA NPs were prepared using the emulsion solvent diffusion method in water by Hosokawa Micron Corp (Hirakata, Japan), as described previously. ${ }^{19,20}$ The particle surface was modified with chitosan to provide a positive charge and thereby strengthen its electrostatic interaction with the cytoplasmic membrane.

\section{Animal Model}

This study conformed to the Guide for the Care and Use of Laboratory Animals, Eighth Edition prepared by the Institute for Laboratory Animal Research and the Guidelines for Proper Conduct of Animal Experiments issued by the Science Council of Japan, which comply with Notice No 71 of the Ministry of Education, Culture, Sports, Science and Technology of Japan. The Animal Research Committee of Kyoto University Graduate School of Medicine approved all animal care procedures, experiments, and methods on March 25, 2016 (approval reference Med Kyo 16541).

Male Japanese white rabbits weighing 2.7 to $3.0 \mathrm{~kg}$ were maintained in a 12-hout light-dark cycle and fed a regular rabbit chow diet. Animals were anesthetized by intravenously administering pentobarbital sodium ( $25 \mathrm{mg}$ / $\mathrm{kg}$ ) and subcutaneously administering lidocaine hydrochloride $(50 \mathrm{mg} / \mathrm{kg})$. A rabbit intimal hyperplasia model was previously prepared and used, as described elsewhere (Video 1). ${ }^{10}$ After a midline neck incision, a 20- to 30 -mm segment of the right jugular vein was exposed, all the branches of the vein were ligated, and a 2-French Fogarty balloon catheter (Edwards Lifesciences LLC, Irvine, Calif) was inserted. The balloon was inflated with $0.2 \mathrm{~mL}$ of air, and the intima of the vein was denuded using 3 passages of the catheter for endothelial exfoliation, ${ }^{21}$ which was considered to be

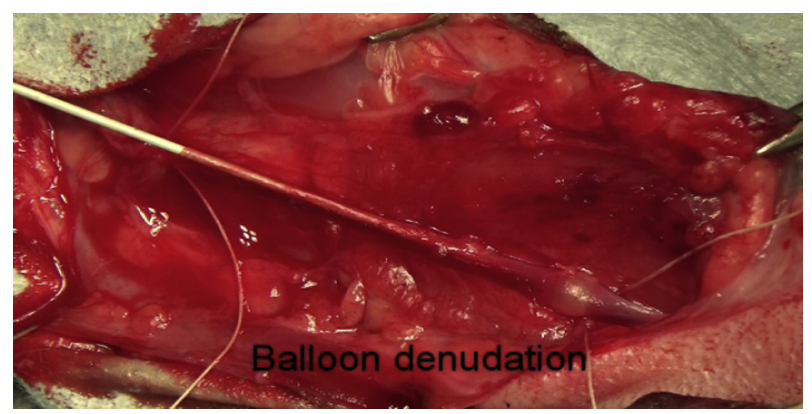

VIDEO 1. Surgical procedure of the rabbit intimal hyperplasia model. Video available at: https://www.jtcvs.org/article/S0022-5223(18)32515-7/fulltext. 
equivalent to distending a saphenous vein graft, and causes endothelial exfoliation. ${ }^{22}$; The rabbits were divided into 3 groups. After removal, the vein grafts from the rabbits were immersed in phosphate-buffered saline (PBS), $0.5 \mathrm{mg} / \mathrm{mL}$ of cont-miR-loaded PLGA NP solution, or $0.5 \mathrm{mg} /$ $\mathrm{mL}$ of miR-145-loaded PLGA NP solution ( $\mathrm{n}=8$ for each), all at room temperature for 30 minutes. After the intravenous administration of heparin $(200 \mathrm{IU} / \mathrm{kg})$, the ipsilateral carotid artery was replaced with the vein graft in reversed end-to-end fashion. Anastomoses were accomplished using 8-0 polypropylene interrupted sutures. To facilitate intimal hyperplasia and simulate a poor runoff condition, the internal carotid artery was ligated with 4-0 silk sutures and the wound was closed layer by layer. Prophylactic cefazolin sodium was administered intravenously. None of the rabbits suffered from central nervous system disorders, including hemiplegia, which could be attributed to the well developed circle of Willis and the posterior circulation consisting of the vertebral and basilar arteries, which are capable of receiving the full load of blood flow. ${ }^{23,24}$ Two weeks after implantation, the vein grafts were explanted under general and local anesthesia, and the rabbits were euthanized using an overdose of sodium pentobarbital.

\section{Intimal Hyperplasia Assessment}

To verify cellular uptake of the PLGA NPs (Figure 1, A), vein grafts were excised and soaked in $0.5 \mathrm{mg} / \mathrm{mL}$ of FITC-loaded PLGA NP solution or PBS for 30 minutes. Fluorescence was detected and visualized from the vein grafts using an all-in-one fluorescence microscope (BZ-9000; Keyence, Osaka, Japan).

The harvested vein grafts were immersed in $4 \%$ paraformaldehyde for 48 hours. Four sections were obtained from each vein graft and stained with hematoxylin and eosin and Elastica Van Gieson. The neointima was defined as the area from the inner surface to the internal elastic lamina. The cross-sectional intimal and medial thicknesses were equiangularly measured at 8 views per section, and the average of these 8 values was used as intimal and medial thicknesses, respectively. The cross-sectional intima/media thickness ratio was also calculated. Each measurement was repeated 3 times. All images were acquired on an all-in-one microscope
(BZ-9000; Keyence). Measurements were made using ImageJ software (version 1.50i, National Institutes of Health, Bethesda, Md). ${ }^{25}$

\section{Statistical Analysis}

Data are presented as mean \pm standard deviation and were analyzed using GraphPad Prism software (version 7.04; GraphPad Software Inc, San Diego, Calif). Data were analyzed using the Welch $t$ test or 1-way analysis of variance followed by the Tukey-Kramer multiple comparisons test. Differences were considered significant at $P<.05$.

\section{RESULTS}

\section{Transduced miR-145 Attenuates Smooth Muscle Cell Proliferating Activity In Vitro}

To examine whether miR-145 is transduced and capable of affecting the phenotypic modulation of smooth muscle cells in an in vitro setting, we measured the miR-145 expression using quantitative polymerase chain reaction (PCR) and quantified the number of proliferating cells using Ki-67 immunocytochemical staining. The miR-145 expression was 18.5 -fold higher in the miR-145-treated group (Figure 2, $A$; miR-145-treated vs cont-miR-treated group; $47.3 \pm 6.5$ vs $2.6 \pm 2.0 ; P<.01 ; \mathrm{n}=3$ each). The results of Ki-67-immunocytochemical analysis revealed that the ratio of $\mathrm{Ki}-67$-positive cells to the total cells counted was lower in the miR-145-treated group (Figure 2, $B$; miR-145-treated vs cont-miR-treated group; $17.5 \%$ and $13.1 \%$ vs $36.2 \%$ and $29.3 \% ; \mathrm{n}=2$ each). These results indicate that miR-145 was incorporated into the smooth muscle cells and was expressed to reduce the proliferating activity of smooth muscle cells in an in vitro setting.

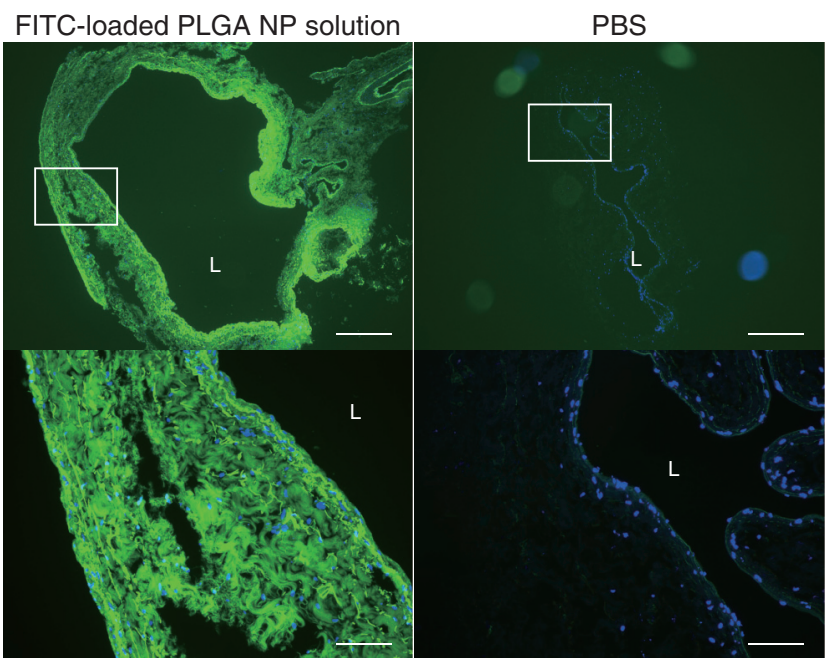

A

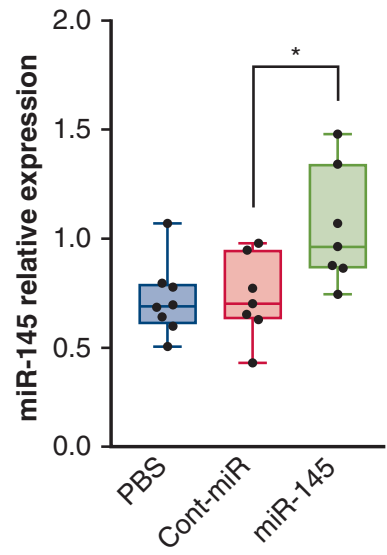

B

FIGURE 1. Fluorescein isothiocyanate (FITC)-loaded poly(lactic-co-glycolic acid) (PLGA) nanoparticles (PLGA NPs) integrate into the cytoplasm, and microRNA-145 (miR-145)-loaded PLGA NPs increase miR-145 expression in vivo. A, Fluorescence microscopy images of rabbit jugular veins immersed in FITC-loaded PLGA NP solution or phosphate-buffered saline (PBS) for 30 minutes. Scale bars $=500 \mu \mathrm{m}$ (upper) and $100 \mu \mathrm{m}$ (lower). Blue denotes nuclei counterstained by 4',6-diamidino-2-phenylindole. $L$ indicates vascular lumen. B, MiR-145 expression level was evaluated 2 weeks after implantation using quantitative polymerase chain reaction. It was normalized to U6 expression and compared among PBS-treated $(\mathrm{n}=8)$, control-miR $($ cont-miR)-treated $(\mathrm{n}=7)$, and miR-145-treated $(\mathrm{n}=7)$ groups. $* P<.05$ versus the cont-miR-treated group. 


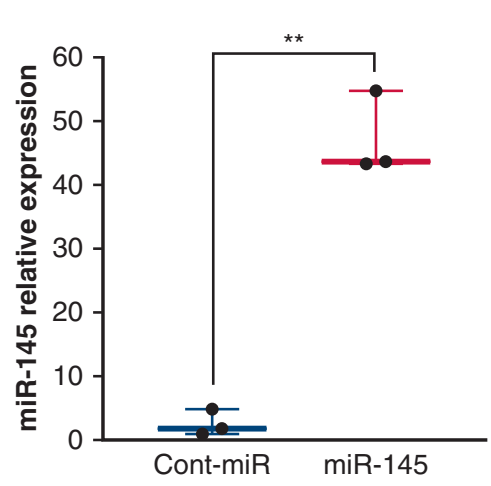

A

FIGURE 2. MicroRNA-145 ( $m i R-145)$ is incorporated into the smooth muscle cells and is expressed to reduce the proliferating activity of smooth muscle cells in an in vitro setting. A, MiR-145 expression level was evaluated 2 days after transduction using quantitative polymerase chain reaction. MiR-145 expression was normalized to U6 expression, and then control-miR (cont-miR)-treated $(\mathrm{n}=3$ ) and miR-145-treated ( $\mathrm{n}=3$ ) groups were compared. $* * P<.01$. B, Representative Ki-67 immunostaining micrographs of the cultured smooth muscle cells from the 2 groups, 2 days after transduction. Scale bars $=100 \mu \mathrm{m}$. Blue denotes nuclei counterstained by 4',6-diamidino-2-phenylindole (DAPI).

\section{Efficient miR-145 Transduction to Vein Grafts Through the PLGA NP System}

To confirm the penetration of PLGA NPs through the cell membrane and the integration into the cytoplasm, we performed the distribution kinetics assay using FITCloaded PLGA NPs. Figure 1, A, shows that the green fluorescence was detected in a vein graft immersed in an FITC-loaded PLGA NP solution whereas green fluorescence was not detected in that soaked in PBS. In the vein graft soaked with FITC-loaded PLGA NP solution, the fluorescence was observed within the cytoplasm and was spread uniformly throughout the vein wall, indicating successful transduction of agent to the vein grafts using this PLGA NP system.

In the rabbit intimal hyperplasia models, the miR-145 expression level was evaluated in the vein grafts 2 weeks after implantation. The results of quantitative PCR revealed a significantly higher miR-145 expression in vein grafts soaked in the miR-145-loaded PLGA NP solution compared with those soaked in the cont-miRloaded PLGA NP solution (Figure 1, B, PBS-treated vs cont-miR-treated vs miR-145-treated group; $0.69 \pm 0.17$ vs $0.70 \pm 0.19$ vs $1.03 \pm 0.28$, respectively; $P=.03$ for the miR-145-treated vs the cont-miR-treated groups). These results indicate that miR-145 can be successfully introduced into vein grafts through the PLGA NP system.

\section{MiR-145-Loaded PLGA NPs Attenuate Intimal Hyperplasia}

To ascertain the effects of miR-145-loaded PLGA NPs on intimal hyperplasia, neointimal area and thickness were measured and compared, respectively. As shown in Figure 3, A, whereas neointimal thickness increased in the PBS-treated and the cont-miR-treated vein grafts at 2 weeks after the procedure, neointimal thickening was suppressed in the miR-145-treated vein graft. We performed quantitative analyses for the intimal/medial area and thickness (Figure 3, B). The cross-sectional area measurement showed that the intimal area in the miR-145-treated group was significantly lower than that of the cont-miR-treated group (PBS-treated vs cont-miR-treated vs miR-145-treated group; $1.63 \pm 0.52 \mathrm{~mm}^{2}$ vs $1.67 \pm 0.49 \mathrm{~mm}^{2}$ vs $0.88 \pm 0.34 \mathrm{~mm}^{2}$, respectively; $P<.01$ for the miR145-treated vs the cont-miR-treated groups). Analyses of the intimal thickness and the intima/media thickness ratio showed a tendency similar to that observed with neointimal area (intimal thickness, $176.8 \pm 23.5 \mu \mathrm{m}$ vs $184.8 \pm 22.4 \mu \mathrm{m}$ vs $88.0 \pm 26.1 \mu \mathrm{m}$, respectively; $P<.01$ for the miR-145-treated vs the cont-miR-treated groups; and intima/media thickness ratio: $1.90 \pm 0.29 \mathrm{vs}$ $1.79 \pm 0.23$ vs $0.89 \pm 0.28$, respectively; $P<.01$ for the miR-145-treated vs the cont-miR-treated groups). These results suggest that the ex vivo microRNA delivery using miR-145-loaded PLGA NPs can reduce intimal hyperplasia.

\section{MiR-145-Loaded PLGA NPs Preserve the Contractile Phenotype of VSMCs}

Next, we evaluated the effects of miR-145-loaded PLGA NPs on VSMC phenotype, which changes from a contractile state to a proliferative (dedifferentiated) state along with neointimal hyperplasia. Figure $4, A$, shows that the ratio of $\mathrm{Ki}-67$-positive proliferative cells in the neointima was lower in the miR-145-treated grafts than those in the 


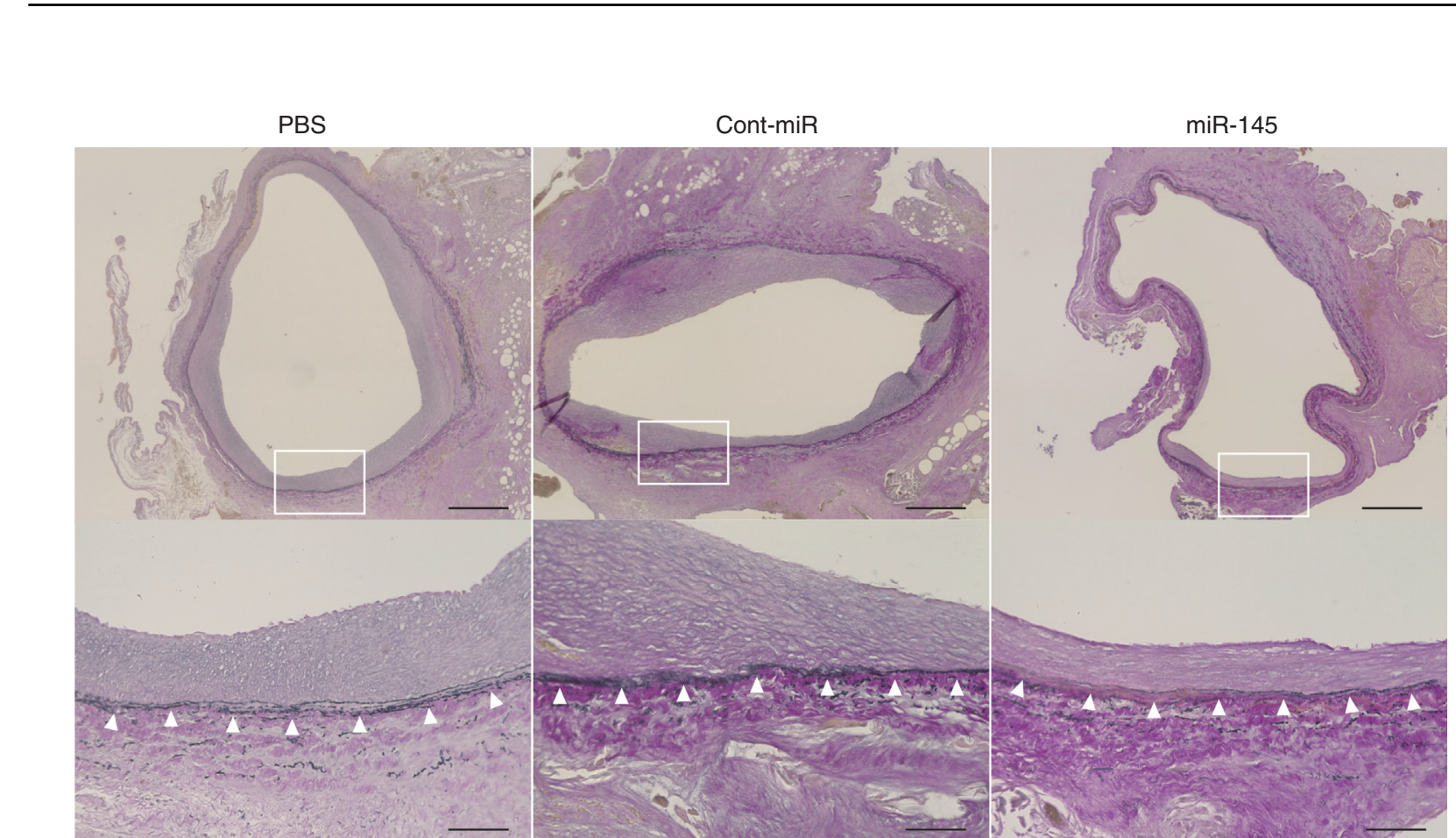

A
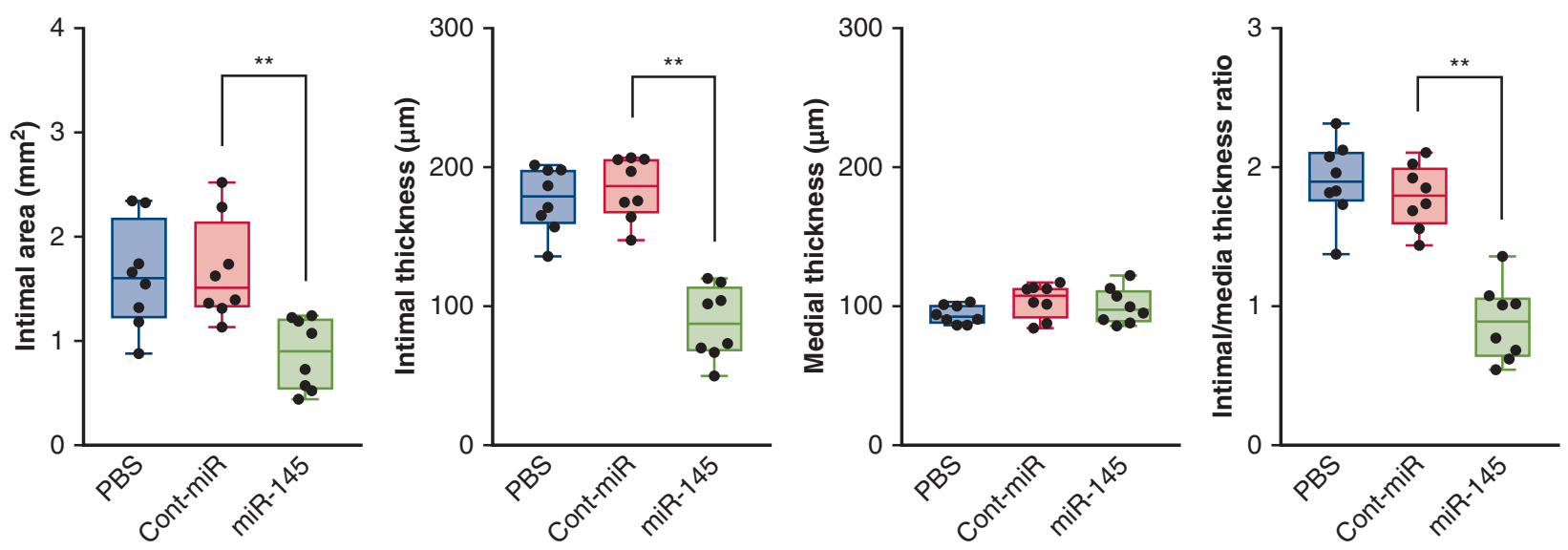

B

FIGURE 3. MicroRNA-145 (miR-145)-loaded poly(lactic-co-glycolic acid) nanoparticles reduce intimal hyperplasia. A, Representative micrographs of cross-sections of phosphate-buffered saline $(P B S)$-treated, control-miR (cont-miR)-treated, or miR-145-treated vein grafts 2 weeks after implantation, stained with Elastica Van Gieson $(\mathrm{n}=8$ each). White arrowheads indicate the internal elastic lamina. Scale bars $=500 \mu \mathrm{m}($ upper $)$ and $100 \mu \mathrm{m}($ lower $)$. $\mathrm{B}$, Intimal area, intimal thickness, medial thickness, and intima/media thickness ratio for each sample from the 3 groups are quantified. $* * P<.01$ versus the cont-miR-treated group.

cont-miR-treated grafts. Additionally, compared with the other 2 groups, quantitative analysis of Ki-67 staining revealed a significantly lower ratio of $\mathrm{Ki}-67$-positive cells in the miR-145-treated group (Figure 4, B; PBS-treated vs cont-miR-treated vs miR-145-treated group: $42.5 \% \pm 7.3 \%$ vs $42.2 \% \pm 6.7 \%$ vs $28.9 \% \pm 4.1 \%$, respectively; $P<.01$ for the miR-145-treated vs the contmiR-treated groups; $\mathrm{n}=6$ for each), indicating that miR145-loaded PLGA NPs reduced VSMC proliferation in the neointima.
Myocardin and Kruppel-like factor 4 (KLF4) are reported to be direct targets of miR-145 and are associated with VSMC differentiation. ${ }^{9}$ MiR-145 regulates myocardin to promote VSMC differentiation, and KLF4 to suppress differentiation. Quantitative real-time PCR analysis showed that mRNA expression of myocardin was significantly upregulated in the miR-145-treated group (Figure 4, C; $0.58 \pm 0.67$ vs $0.61 \pm 0.46$ vs $1.70 \pm 0.72$, respectively; $P=.02$ for the miR-145-treated vs the cont-miR-treated groups; $\mathrm{n}=6$ for each). In contrast, KLF4 expression was 


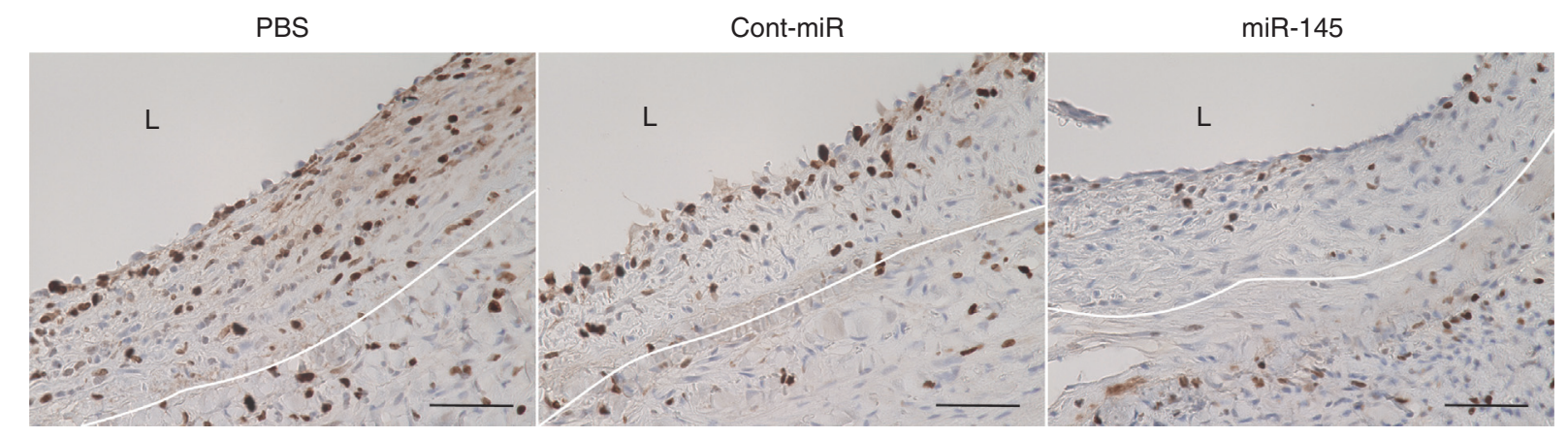

A

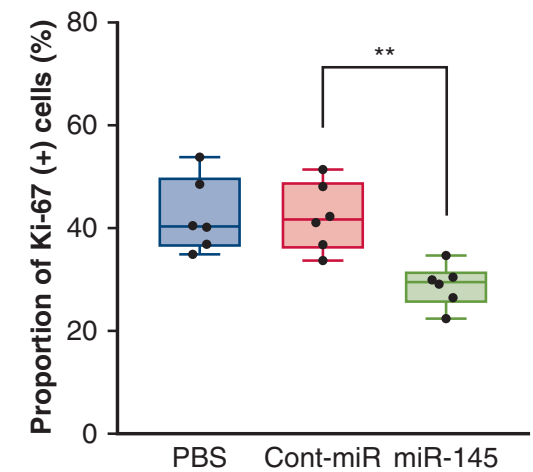

B

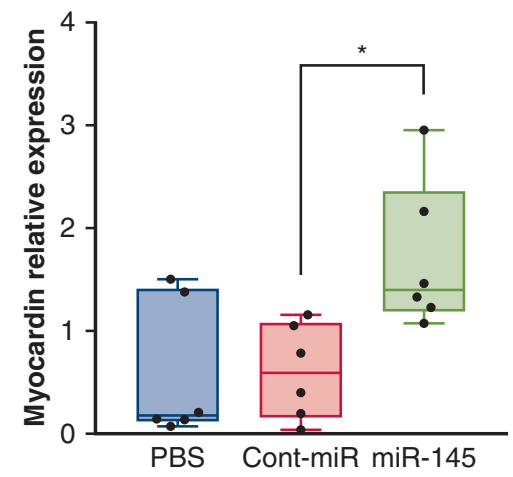

C

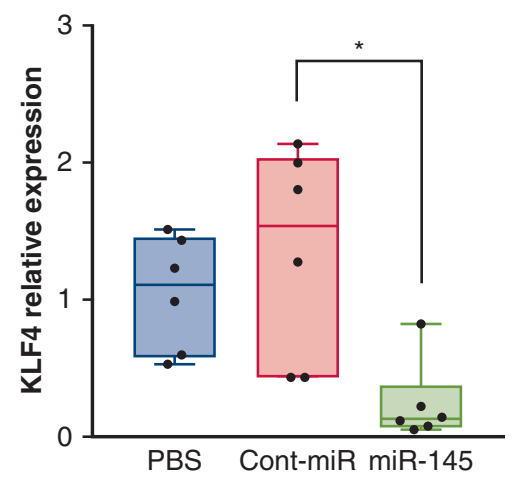

D

FIGURE 4. MicroRNA-145 (miR-145)-loaded poly(lactic-co-glycolic acid) nanoparticles preserve the contractile phenotype of vascular smooth muscle cells. A, Representative Ki-67 immunostaining micrographs of the vein grafts from the 3 groups, 2 weeks after implantation. White lines correspond to the internal elastic lamina. Scale bars $=40 \mu \mathrm{m}$. L indicates vascular lumen. B, Ratio of the number of Ki-67-positive cells to total cell numbers in the intima of the vein grafts from the groups are compared $(\mathrm{n}=6$ each). C and $\mathrm{D}$, The expression level of miR-145 target genes, myocardin (C), and Kruppel-like factor 4 (KLF4) (D) in the vein wall from the 3 groups ( $\mathrm{n}=6$ each), 2 weeks after implantation, were evaluated using quantitative polymerase chain reaction, with expression normalized to corresponding glyceraldehyde-3-phosphate dehydrogenase expression. $* P<.05$, ** $P<.01$ versus the control-miR (cont-miR)treated group. PBS, Phosphate-buffered saline.

significantly downregulated in the miR-145-treated group (Figure $4, D ; 1.05 \pm 0.42$ vs $1.35 \pm 0.77$ vs $0.24 \pm 0.29$, respectively; $P<.01$ for the miR-145-treated vs the contmiR-treated groups; $\mathrm{n}=6$ for each). These findings indicate that the overexpression of miR-145 might have preserved contractile and differentiated VSMC phenotype through the regulation of myocardin and KLF4.

\section{MiR-145-Loaded PLGA NPs Modulate the Expression of Inflammation-Related Molecules}

Intimal hyperplasia is caused by specific mechanisms such as VSMC phenotypic modulation and inflammation. ${ }^{2}$ It is reported that the expression level of CD40, which is known as a putative direct target of miR-145 because of its nucleotide sequence complementarity (Figure 5, A), is associated with intimal hyperplasia during the acute inflammation phase through the nuclear factor-kappa B $(\mathrm{NF}-\kappa \mathrm{B})$-dependent pathway. ${ }^{26,27}$ To assess the effects of miR-145-loaded PLGA NPs on inflammation, CD40 and $\mathrm{NF}-\kappa \mathrm{B}$ protein expression levels were measured using
Western blot analysis, which revealed that CD40 expression was significantly reduced in the miR-145-treated group (Figure 5, $B$ and $C$; PBS-treated vs cont-miRtreated vs miR-145-treated group; $0.61 \pm 0.18$ vs $0.66 \pm 0.17$ vs $0.22 \pm 0.16$, respectively; $P<.01$ for the miR-145-treated vs the cont-miR-treated groups; $\mathrm{n}=6$ for each). NF- $\kappa \mathrm{B}$ protein levels also showed a similar pattern of expression level (Figure 5, $B$ and $C$; $0.68 \pm 0.19$ vs $0.68 \pm 0.17$ vs $0.31 \pm 0.18$, respectively; $P<.01$ for the miR-145-treated vs the cont-miR-treated groups; $\mathrm{n}=6$ for each). These data suggest that miR145-loaded PLGA NPs can suppress the inflammation processes through regulation of the expression of CD40 and $\mathrm{NF}-\kappa \mathrm{B}$.

\section{DISCUSSION}

The results presented in the present study show that the ex vivo miR-145 delivery system using PLGA NPs, a controlled release system of microRNAs without viral or plasmid vectors, can efficiently limit neointimal 
CD40 3'-UTR

(Homo sapiens; human)

(Oryctolagus cuniculus; rabbit)

hsa-miR-145-5p (human)

ocu-miR-145-5p (rabbit)

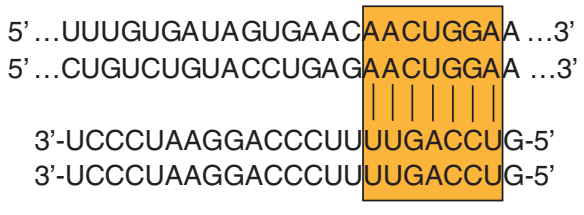

A
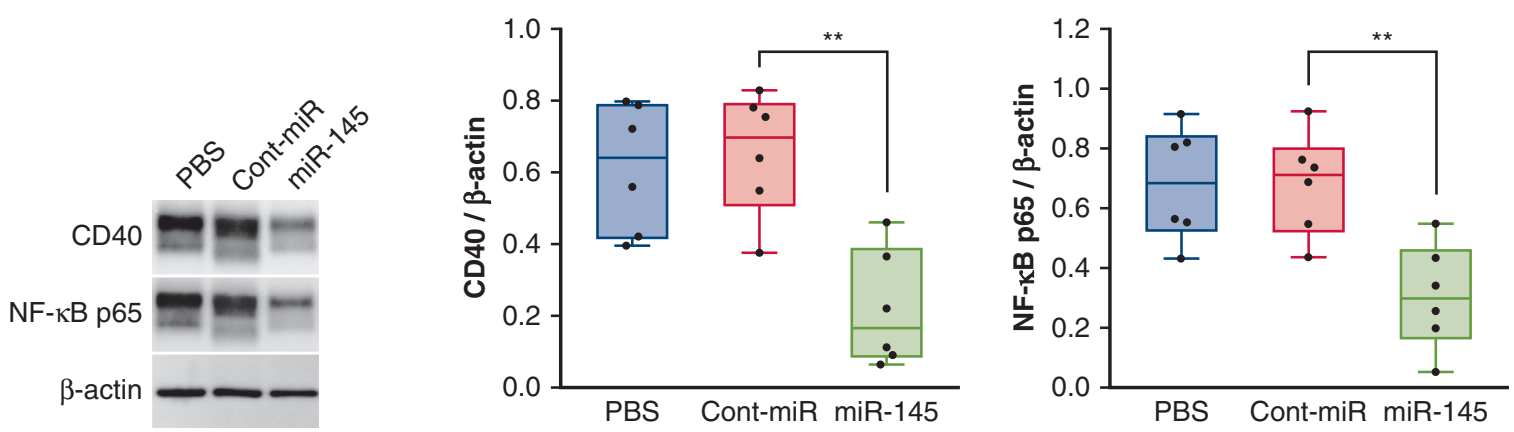

B

C

FIGURE 5. MicroRNA-145 (miR-145)-loaded poly(lactic-co-glycolic acid) nanoparticles modulate the expression of inflammation-related molecules. A, The putative miR-145 binding sequence of CD40 3'-untranslated region (UTR). B, Representative Western blots for CD40 and nuclear factor-kappa B (NF$\kappa B$ ) expression in the vein wall from the 3 groups, 2 weeks after implantation. C, Semiquantitative results of CD40 and NF- $\kappa$ B in the vein wall from the 3 groups $(\mathrm{n}=6$ each); protein expression was normalized to $\beta$-actin expression. $* * P<.01$ versus the control-miR (cont-miR)-treated group. $P B S$, Phosphatebuffered saline.

hyperplasia of vein grafts by regulating the phenotypic modulation of VSMCs (Figure 6). This simple ex vivo miR-145-loaded PLGA NP delivery system holds advantages in clinical settings.
Cellular uptake of microRNA duplexes through the plasma membrane is an obstacle for gene transduction. Various strategies have been designed to overcome the problem such as antibody-mediated delivery, cholesterol
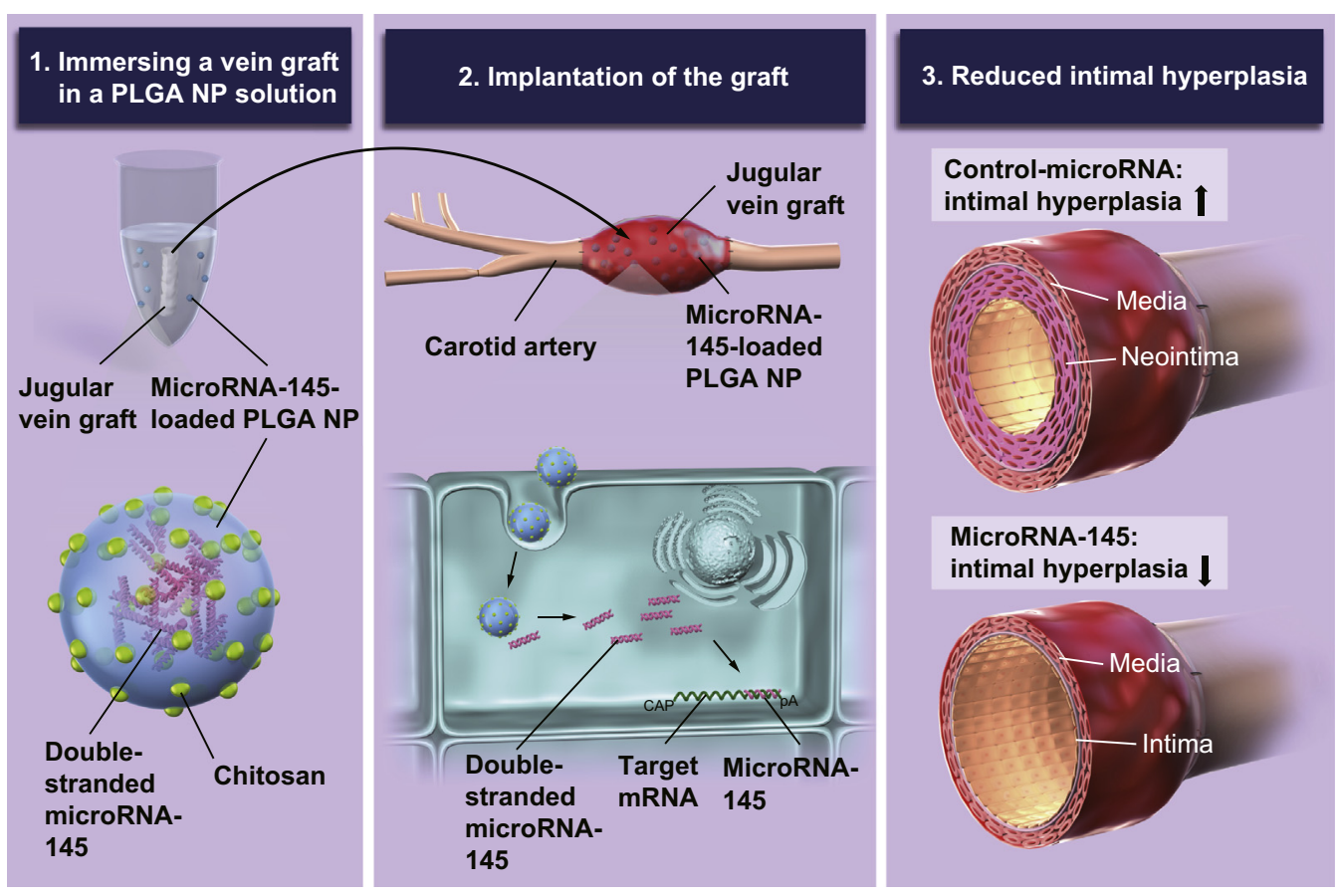

FIGURE 6. Schematic summary of the present study. PLGA, poly(lactic-co-glycolic acid); $N P$, nanoparticle. 
conjugation, hydrodynamic injection, transfection agents, viral vectors, and electroporation. ${ }^{28,29}$ Most studies that have shown the inhibitory effect of miR-145 on neointimal hyperplasia using animal disease models have commonly used viral vectors to introduce miR-145 into the cytoplasm. $^{30-32}$ Although there have been a number of developments in the viral delivery system so far, the risk for oncogenicity and immunogenicity remain as major concerns.

To address those issues, we have used an ex vivo gene transfer system without viral or plasmid vectors using PLGA NPs. Previous studies have described that decreasing the dimension of particles results in higher uptake efficiency. ${ }^{13,33}$ Furthermore, recognizing that PLGA polymers containing a 50:50 ratio of lactic and glycolic acids are hydrolyzed rapidly, we used a ratio of $75 \%$ lactide to $25 \%$ glycolide to prolong nanoparticle half-life to 2 weeks in the present study. ${ }^{34}$ Customizing the component and size, we optimized the formulation of PLGA NPs suitable for the sustained and efficient delivery of miR-145 to the vein grafts.

Local delivery is a promising therapeutic option of this ex vivo gene transfer system because it can circumvent offtarget effects. The Project of Ex-vivo Vein Graft Engineering via Transfection (PREVENT) IV trial was one of the representative phase III randomized trials in which saphenous vein grafts were treated ex vivo with an E2F transcription factor oligodeoxynucleotide decoy, edifoligide, in a pressure-mediated method during coronary artery bypass graft surgery. ${ }^{6}$ The authors mentioned that the high rates of short-term vein graft failure could be attributed to the pressure-mediated delivery system. Furthermore, a previous basic study has described that immersing a jugular vein graft in an imatinib mesylate-incorporated PLGA nanoparticle solution for 30 minutes resulted in the suppression of the neointimal formation in its rabbit vein graft implantation model ${ }^{35}$ On the basis of the findings of the clinical trial and the basic study, the vein grafts in the present study were soaked in a solution containing the nanoparticles for 30 minutes rather than following the pressure-mediated method. The novel efficiency of transduction was confirmed by the fluorescence detection after the treatment with FITCloaded PLGA NPs and the increased expression level of miR-145 by the miR-145-loaded PLGA NP treatment.

We showed a significant suppressive effect of miR-145 on neointimal hyperplasia. Immunohistochemical analyses revealed that miR-145-loaded PLGA NPs decreased the number of Ki-67-positive proliferating cells, suggesting that miR-145 in our model system attenuated phenotypic modulation of VSMCs. Vein graft diseases develop through 3 traditionally classified steps: thrombosis, intimal hyperplasia, and atherosclerosis. ${ }^{3}$ When surgically harvested, saphenous veins are exposed to hypoxia and malnutrition, which initiate acute inflammatory responses after implantation.
When implanted into an arterial environment, the vein grafts are subjected to stretching forces and shear stress, causing intimal hyperplasia. At this stage, VSMCs play several important roles by altering their biological properties through a process known as phenotypic modulation. ${ }^{36,37}$ After the thrombosis and intimal hyperplasia, lipid accumulation takes place at the intima, and finally causes atherosclerosis. The regulation of the deterioration of vein graft disease progressing as a series of chronological events would be anticipated as a therapeutic strategy.

We set the time frame of 2 weeks after the implantation of vein grafts considering the working duration of gene-loaded PLGA NPs and the optimal time point to evaluate the intimal hyperplasia and VSMC phenotypic modulation. The chronological change of the intimal hyperplasia and VSMC proliferating activity have been previously studied in a rabbit jugular vein implantation model, in which the intimal thickening began at 2 weeks after implantation and remained constant after 12 weeks. ${ }^{38}$ The investigators have reported that the VSMC proliferating activity reached a peak at 1 week after implantation and gradually decreased thereafter, concluding that the increase in the wall thickening after 4 weeks was mainly due to the production of extracellular matrix. In contrast, an in vitro experiment using PLGA NPs has shown that the gene expression, which was derived from the plasmid DNA-loaded PLGA NPs, was constantly detected until the sixth day after transduction but declined to the level of one-tenth on the ninth day. ${ }^{39}$ On the basis of these findings, we considered the 2-week observational period to be valid to fundamentally assess the effect of the miR-145-loaded PLGA NPs.

CD40 is a membrane protein receptor, which belongs to the tumor necrosis factor receptor superfamily and is expressed in inflammatory cells, as well as in vascular endothelial or smooth muscle cells. ${ }^{40,41}$ The activation of CD40 stimulates the downstream signaling pathway, such as the NF- $\kappa \mathrm{B}$ pathway, which subsequently upregulates proinflammatory and proatherogenic genes. ${ }^{42}$ Song and colleagues have reported that vascular wall cell-derived CD40 plays a role in the vascular response to injury. ${ }^{26}$ Furthermore, recent studies have identified CD40 as a putative direct target of miR-145 because of its nucleotide sequence complementarity, and miR-145 has been reported to repress VSMC proliferation by targeting CD $40 .{ }^{27}$ We show that the overexpression of miR-145 reduced protein levels of CD40 and NF- $\kappa \mathrm{B}$ and that intimal hyperplasia is likely to be associated with CD40 and NF- $\kappa$ B expression. Further studies should be conducted to clarify the underlying mechanisms.

\section{Limitations}

There are several limitations to our study. First, we have not conducted dose-effect relationship studies between the concentration of PLGA NP solution and cellular uptake although a previous study showed that the uptake of PLGA 
NPs reached a saturation limit at a concentration of $0.5 \mathrm{mg} /$ $\mathrm{mL}{ }^{33}$ The evaluation of a dose-dependent effect and preclinical safety tests should be performed before the clinical application of the present strategy. Second, the suppressive efficiency of our technique in the intimal hyperplasia was not compared with those of viral- or plasmid-mediated treatments. Previous studies using miR-145 as a therapeutic agent with viruses or plasmids have shown approximately a $50 \%$ reduction in the intimal thickness at 2 weeks after treatment, which is equivalent to that in the present study. ${ }^{10,30-32}$ Although those studies vary in terms of animal species treated, transduction methods, and/or measured variables, the comparison of the results among previous and present studies might suggest that our technique would not be less efficient than previously reported methods. However, a comparative study using viruses, plasmids, and PLGA nanoparticles in the same condition is warranted to verify the efficiency of PLGA nanoparticles in the suppression of intimal hyperplasia. Third, the rabbit jugular vein implantation model used in the present study was not fully assumed as a coronary artery bypass grafting model, but as a peripheral artery bypass grafting model. Moreover, carotid atherosclerosis is not identical to coronary atherosclerosis. Thus, assuming this technique as a new treatment option for ischemic heart disease, efficacy of this treatment in more applicable models, such as an animal coronary artery bypass grafting model, should be verified. Finally, the long-term effect of miR-145-loaded PLGA NPs on vein graft diseases was not fully evaluated. Although the efficiency of miR-145-loaded PLGA NPs theoretically disappear at 4 weeks after implantation, whether attenuating the inflammation and VSMC phenotypic modulation in the initial period affects the long-term suppression of intimal thickening remains unknown. Therefore, a long-term study should include the evaluation of the extracellular matrix production, which mainly accounts for the intimal thickening after 4 weeks of implantation. Considering clinical application, it is crucial to evaluate the long-term results recapitulating bona fide etiology of the progression of human vein graft diseases occurring through thrombosis, intimal hyperplasia, and atherosclerosis over a period of decades.

\section{CONCLUSIONS}

The local sustained release of miR-145 using PLGA NPs attenuated intimal hyperplasia by maintaining the VSMCs in a contractile state. Considering the simple maneuverability in an operating room setting, our ex vivo miR-145 delivery system appears to be promising for clinical applications in the future.

\section{Conflict of Interest Statement}

Authors have nothing to disclose with regard to commercial support.
The authors thank Mr Shuichi Miyake (Kyoto University) for the technical support and Dr Hemant Poudyal (Kyoto University) for critical reading of the manuscript.

\section{References}

1. Goldman S, Zadina K, Moritz T, Ovitt T, Sethi G, Copeland JG, et al. Long-term patency of saphenous vein and left internal mammary artery grafts after coronary artery bypass surgery: results from a Department of Veterans affairs cooperative study. J Am Coll Cardiol. 2004;44:2149-56.

2. Muto A, Model L, Ziegler K, Eghbalieh SD, Dardik A. Mechanisms of vein graft adaptation to the arterial circulation: insights into the neointimal algorithm and management strategies. Circ J. 2010;74:1501-12.

3. Motwani JG, Topol EJ. Aortocoronary saphenous vein graft disease: pathogenesis, predisposition, and prevention. Circulation. 1998;97:916-31.

4. Shachner T. Pharmacologic inhibition of vein graft neointimal hyperplasia. $J$ Thorac Cardiovasc Surg. 2006;131:1065-72.

5. Banno H, Takei Y, Muramatsu T, Komori K, Kadomatsu K. Controlled release of small interfering RNA targeting midkine attenuates intimal hyperplasia in vein grafts. J Vasc Surg. 2006;44:633-41.

6. Alexander JH, Hafley G, Harrington RA, Peterson ED, Ferguson TB Jr, Lorenz TJ, et al. Efficacy and safety of edifoligide, an E2F transcription factor decoy, for prevention of vein graft failure following coronary artery bypass graft surgery: PREVENT IV: a randomized controlled trial. JAMA. 2005;294:2446-54.

7. Calin GA, Croce CM. MicroRNA signatures in human cancers. Nat Rev Cancer. 2006;6:857-66.

8. O'Sullivan JF, Martin K, Caplice NM. Microribonucleic acids for prevention of plaque rupture and in-stent restenosis: "a finger in the dam." J Am Coll Cardiol. 2011;57:383-9.

9. Cordes KR, Sheehy NT, White MP, Berry EC, Morton SU, Muth AN, et al. miR145 and miR-143 regulate smooth muscle cell fate and plasticity. Nature. 2009; 460:705-10.

10. Ohnaka M, Marui A, Yamahara K, Minakata K, Yamazaki K, Kumagai M, et al Effect of microRNA-145 to prevent vein graft disease in rabbits by regulation of smooth muscle cell phenotype. J Thorac Cardiovasc Surg. 2014;148:676-82.

11. Athanasiou KA, Niederauer GG, Agrawal CM. Sterilization, toxicity, biocompatibility and clinical applications of polylactic acid/polyglycolic acid copolymers. Biomaterials. 1996;17:93-102.

12. Lü JM, Wang X, Marin-Muller C, Wang H, Lin PH, Yao Q, et al. Current advances in research and clinical applications of PLGA-based nanotechnology. Expert Rev Mol Diagn. 2009;9:325-41.

13. Panyam J, Labhasetwar V. Biodegradable nanoparticles for drug and gene deliv ery to cells and tissue. Adv Drug Deliv Rev. 2003;55:329-47.

14. Stein CA, Cheng YC. Antisense oligonucleotides as therapeutic agents-is the bullet really magical? Science. 1993;261:1004-12.

15. Soutschek J, Akinc A, Bramlage B, Charisse K, Constien R, Donoghue M, et al Therapeutic silencing of an endogenous gene by systemic administration of modified siRNAs. Nature. 2004;432:173-8.

16. Kim DH, Behlke MA, Rose SD, Chang MS, Choi S, Rossi JJ. Synthetic dsRNA Dicer substrates enhance RNAi potency and efficacy. Nat Biotechnol. 2005;23: 222-6.

17. Izuhara M, Kuwabara Y, Saito N, Yamamoto E, Hakuno D, Nakashima T, et al. Prevention of neointimal formation using miRNA-126-containing nanoparticleconjugated stents in a rabbit model. PLoS One. 2017;12:e0172798.

18. Tsumaru S, Masumoto H, Minakata K, Izuhara M, Yamazaki K, Ikeda T, et al. Therapeutic angiogenesis by local sustained release of microRNA-126 using poly lactic-co-glycolic acid nanoparticles in murine hindlimb ischemia. $J$ Vasc Surg. 2018;68:1209-15.

19. Tahara K, Sakai T, Yamamoto H, Takeuchi H, Kawashima Y. Establishing chitosan coated PLGA nanosphere platform loaded with wide variety of nucleic acid by complexation with cationic compound for gene delivery. Int J Pharm. 2008; 354:210-6.

20. Tahara K, Samura S, Tsuji K, Yamamoto H, Tsukada Y, Bando Y, et al. Oral nuclear factor- $\kappa \mathrm{B}$ decoy oligonucleotides delivery system with chitosan modified poly(D,L-lactide-co-glycolide) nanospheres for inflammatory bowel disease. Biomaterials. 2011;32:870-8.

21. Davies MG, Dalen H, Svendsen E, Hagen PO. Influence of perioperative catheter injury on the long-term vein graft function and morphology. J Surg Res. 1996;66: 109-14.

22. Dashwood MR, Savage K, Tsui JC, Dooley A, Shaw SG, Fernández Alfonso MS, et al. Retaining perivascular tissue of human saphenous vein grafts protects 
against surgical and distension-induced damage and preserves endothelial nitric oxide synthase and nitric oxide synthase activity. J Thorac Cardiovasc Surg. 2009; 138:334-40

23. Lehman RM, Owens GK, Kassell NF, Hongo K. Mechanism of enlargement of major cerebral collateral arteries in rabbits. Stroke. 1991;22:499-504.

24. Tutino VM, Mandelbaum M, Choi H, Pope LC, Siddiqui A, Kolega J, et al. Aneurysmal remodeling in the circle of Willis after carotid occlusion in an experimental model. J Cereb Blood Flow Metab. 2014;34:415-24.

25. Schneider CA, Rasband WS, Eliceiri KW. NIH Image to ImageJ: 25 years of image analysis. Nat Methods. 2012;9:671-5.

26. Song Z, Jin R, Yu S, Nanda A, Granger DN, Li G. Crucial role of CD40 signaling in vascular wall cells in neointimal formation and vascular remodeling after vascular interventions. Arterioscler Thromb Vasc Biol. 2012;32:50-64.

27. Guo X, Li D, Chen M, Chen L, Zhang B, Wu T, et al. miRNA-145 inhibits VSMC proliferation by targeting CD40. Sci Rep. 2016;6:35302.

28. Song E, Zhu P, Lee SK, Chowdhury D, Kussman S, Dykxhoorn DM, et al. Antibody mediated in vivo delivery of small interfering RNAs via cell-surface receptors. Nat Biotechnol. 2005;23:709-17.

29. White PJ. Barriers to successful delivery of short interfering RNA after systemic administration. Clin Exp Pharmacol Physiol. 2008;35:1371-6.

30. Cheng Y, Liu X, Yang J, Lin Y, Xu DZ, Lu Q, et al. MicroRNA-145, a novel smooth muscle cell phenotypic marker and modulator, controls vascular neointimal lesion formation. Circ Res. 2009;105:158-66.

31. Lovren F, Pan Y, Quan A, Singh KK, Shukla PC, Gupta N, et al. MicroRNA-145 targeted therapy reduces atherosclerosis. Circulation. 2012;126:S81-90.

32. Elia L, Quintavalle M, Zhang J, Contu R, Cossu L, Latronico MV, et al. The knockout of miR-143 and -145 alters smooth muscle cell maintenance and vascular homeostasis in mice: correlates with human disease. Cell Death Differ. 2009; 16:1590-8.

33. Desai MP, Labhasetwar V, Walter E, Levy RJ, Amidon GL. The mechanism of uptake of biodegradable microparticles in Caco-2 cells is size dependent. Pharm Res. 1997;14:1568-73.
34. Miller RA, Brady JM, Cutright DE. Degradation rates of oral resorbable implant (polylactates and polyglycolates): rate modification with changes in PLA/PGA copolymer ratios. J Biomed Mater Res. 1977;11:711-9.

35. Kimura S, Egashira K, Nakano K, Iwata E, Miyagawa M, Tsujimoto H, et al. Local delivery of imatinib mesylate (STI571)-incorporated nanoparticle ex vivo suppresses vein graft neointima formation. Circulation. 2008;118 S65-70.

36. Chamley-Campbell J, Campbell GR, Ross R. The smooth muscle cell in culture. Physiol Rev. 1979;59:1-61.

37. Owens GK. Regulation of differentiation of vascular smooth muscle cells. Physiol Rev. 1995;75:487-517.

38. Zwolak RM, Adams MC, Clowes AW. Kinetics of vein graft hyperplasia: association with tangential stress. J Vasc Surg. 1987;5:126-36.

39. Tahara K, Sakai T, Yamamoto H, Takeuchi H, Hirashima N Kawashima Y. Improvements in transfection efficiency with chitosan modified poly(DL-lactide-co-glycolide) nanospheres prepared by the emulsion solvent diffusion method, for gene delivery. Chem Pharm Bull. 2011;59:298-301.

40. Anand SX, Viles-Gonzalez JF, Badimon JJ, Cavusoglu E, Marmur JD. Membrane-associated CD40L and SCD40L in atherothrombotic disease. Thromb Haemost. 2003;90:377-84.

41. Mach F, Schönbeck U, Sukhova GK, Bourcier T, Bonnefoy JY, Pober JS, et al. Functional CD40 ligand is expressed on human vascular endothelial cells, smooth muscle cells, and macrophages: implications for CD40-CD40 ligand signaling in atherosclerosis. Proc Natl Acad Sci U S A. 1997;94 1931-6.

42. Chen Y, Chen J, Xiong Y, Da Q, Xu Y, Jiang X, et al. Internalization of CD40 regulates its signal transduction in vascular endothelial cells. Biochem Biophys Res Commun. 2006;345:106-17.

Key Words: microRNA, vein graft, drug delivery system 